\title{
Primeiro registro para o Brasil de Maravalia bolivarensis Y. Ono (Pucciniales) parasitando Manilkara sp. (Sapotaceae) ${ }^{1}$
}

\author{
Ronan Gomes Furtado ${ }^{3}$, Helen Maria Pontes Sotão ${ }^{2,3,4}$, Josiane Santana Monteiro ${ }^{3}$ e Fabiano Melo de Brito
}

Recebido: 11.07.2017; aceito: 8.01.2018

\begin{abstract}
First record for Brazil of Maravalia bolivarensis Y. Ono (Pucciniales) parasitizing Manilkara sp. (Sapotaceae)). This study presents a taxonomic treatment of the phytopathogenic fungus Maravalia bolivarensis (Pucciniales) causing rust in plants of Manilkara sp. (Sapotaceae), in the Amapá National Forest, Amapá State, Brazil. Previously known only from Venezuela, this is the first record of $M$. bolivarensis for Brazil, and its original distribution is extended to the Amazon Biome. Morphological descriptions, illustrations of the microstructures, examined material, geographic distribution and taxonomic comments are provided for this species. Additionally, Maravalia sapotae (Mains) Y. Ono was also recorded in the Amapá National Forest and is being referred here for the first time to the state of Amapá. Finally, an identification key including species of the teleomorph genera Achrotelium, Catenulopsora and Maravalia that occur on plants of Sapotaceae in Brazil is presented.
\end{abstract}

Keywords: Amazon, Chaconiaceae, Maravalia sapotae, Pucciniomycetes

RESUMO - (Primeiro registro para o Brasil de Maravalia bolivarensis Y. Ono (Pucciniales) parasitando Manilkara sp. (Sapotaceae)). Apresenta-se um tratamento taxonômico do fungo fitopatógeno Maravalia bolivarensis (Pucciniales) causando ferrugem em plantas do gênero Manilkara (Sapotaceae), na Floresta Nacional do Amapá, no Estado do Amapá. Previamente conhecida apenas para a Venezuela, este é o primeiro registro de M. bolivarensis para o Brasil e sua distribuição original é estendida para o Bioma Amazônia. Descrições morfológicas, ilustrações das microestruturas, material examinado, distribuição geográfica e comentários taxonômicos são fornecidos. Foi também registrada Maravalia sapotae (Mains) Y. Ono para a Floresta Nacional do Amapá, sendo esta a primeira referência da espécie para o Estado do Amapá. Por fim, apresenta-se uma chave de identificação com as espécies dos gêneros teleomorfos Achrotelium, Catenulopsora e Maravalia que ocorrem sobre plantas da família Sapotaceae no Brasil.

Palavras-chave: Amazônia, Chaconiaceae, Maravalia sapotae, Pucciniomycetes

\section{Introdução}

Maravalia Arthur é um gênero de fungos da família Chaconiaceae e ordem Pucciniales (Aime et al. 2006). Caracteriza-se por apresentar télios subepidermais, irrompentes, teliósporos unicelulares, lateralmente livres, pedicelados ou ocasionalmente sésseis, de parede fina, ocasionalmente engrossada no ápice, hialina ou pigmentada, metabasídio produzido por uma expansão apical do probasídio, formando quatro basidiósporos; espermogônio subcuticular do grupo VI, tipo 5 ou 7 (Ono 1984, Cummins \& Hiratsuka 2003).
Atualmente são conhecidas cerca de 36 espécies de Maravalia para as regiões tropicais e subtropicais, ocorrendo sobre hospedeiros de diversas famílias botânicas. Carvalho Jr. \& Hennen (2009) apresentaram uma chave de identificação para as espécies de Maravalia, modificada e adaptada a partir da chave de Ono (1984). Dentre estas, onze espécies são reportadas sobre plantas da família Sapotaceae ( $M$. aulica (Syd. \& P. Syd.) Y. Ono, M. bolivarensis Y. Ono, M. echinulata (Niessl ex Rabenh.) Y. Ono, $M$. fici (Mundk. \& Thirum.) Y. Ono, M. gentilis (Syd. \& P. Syd.) Y. Ono, M. kevorkianii (Cummins) Cummins \&

1. Parte da Dissertação de Mestrado do primeiro Autor

2. Universidade Federal Rural da Amazônia/Museu Paraense Emílio Goeldi, Programa de Pós-Graduação em Ciências Biológicas: Botânica Tropical, Av. Presidente Tancredo Neves, 2501, Bairro Montese, 66.077-901 Belém, PA, Brasil

3. Museu Paraense Emílio Goeldi, Coordenação de Botânica, Av. Presidente Tancredo Neves, 1901, Bairro Montese, 66.077-901 Belém, PA, Brasil

4. Autor para correspondência: helen@museu-goeldi.br 
Y. Hirats., M. lucumae (Dietel) Y. Ono, M. mimusops (Cooke) Y. Ono, M. palaquii (Cummins) Y. Ono, $M$. payenae (Racib.) Y. Ono e M. sapotae (Mains) Y. Ono) (Ono 1984).

A especificidade de hospedeiro das ferrugens de plantas é importante característica para a taxonomia de Pucciniales. Estes fungos podem completar seu ciclo de vida em apenas um hospedeiro (autoécio) ou em dois hospedeiros pertencentes a famílias filogeneticamente distintas (heteroécio) (Figueiredo \& Passador, 2008). Com raras exceções, um estágio do ciclo de uma espécie não ocorre em mais de uma família vegetal, embora a espécie possa ocorrer em mais de um gênero da mesma família.

Na Lista da Flora do Brasil 2020 em construção (2017), onze espécies de Maravalia são referidas parasitando plantas de sete famílias botânicas: Euphorbiaceae (M. sebastianiae J.C. Lindq.); Erythroxylaceae (M. erythroxyli (Viégas) Y. Ono \& J.F. Hennen); Fabaceae (M. amazonensis (F.C. Albuq.) Y. Ono, M. bauhiniicola (Cummins) Y. Ono e M. swartziae Y. Ono.); Peraceae (M. perae A.A. Carvalho \& J.F. Hennen); Rubiaceae (M. manettiae Jørst.); Sapindaceae (M. allophyli Berndt \& F.O. Freire); Sapotaceae (M. lucumae (Dietel) Y. Ono sobre Lucuma Molina, M. palaquii (Cummins) Y. Ono sobre Palaquium Blanco e M. sapotae sobre Manilkara Adans) (Carvalho Jr. \& Hennen 2009, Hennen et al. 2005, Sotão et al. 2013).

Além de espécies de Maravalia, gêneros de Sapotaceae também hospedam outros gêneros de Pucciniales: Chrysophyllum L. parasitado por Uredo chrysophylli Syd. \& P. Syd. e U. crysophyllicola Henn., Mimusops L. com ocorrência de U. confluens Henn., Pouteria Aubl. parasitada por Achrotelium lucumae Cummins e Catenulopsora henneneae Buriticá, e sobre Sapotaceae indeterminada U. agnostoica Speg (Carvalho Jr. \& Hennen 2009, Hennen et al. 2005, Sotão et al. 2013). Entre estas espécies, A. lucumae, $M$. lucumae e $M$. sapotae tem ocorrência registrada para o bioma Amazônia, ocorrendo no Estado do Pará (Lista da Flora do Brasil 2020 em construção 2017).

Como parte de um inventário realizado na Floresta Nacional (Flona) do Amapá (AP), este trabalho tem por objetivo apresentar um tratamento taxonômico com bases morfológicas e registrar a primeira ocorrência de $M$. bolivarensis para o Brasil. É também proposta uma chave de identificação para as espécies de ferrugens que ocorrem no Brasil, sobre plantas da família Sapotaceae.

\section{Material e métodos}

As coletas de plantas com sintomas de ferrugem foram realizadas na Floresta Nacional do Amapá (51 $53^{\prime} 37^{\prime \prime} W$ e $\left.01^{\circ} 06^{\prime} 37^{\prime \prime} \mathrm{N}\right)$, uma unidade de conservação do bioma Amazônia, cuja vegetação predominante é de Floresta Ombrófila Densa, mas que também inclui áreas inundadas como igapós e manchas de vegetação adaptadas aos afloramentos rochosos (IBAMA 2017).

As técnicas de coleta, preservação e identificação adotadas neste trabalho seguiram Cummins \& Hiratsuka (2003). A identificação dos espécimes foi feita com base na identificação da planta hospedeira e nas análises das microestruturas dos fungos. Para observação microscópica do material foram montadas lâminas semipermanentes de soros e esporos em solução de lactoglicerol (água destilada + ácido láctico + glicerina) (Neergaard et al. 2000), posteriormente aquecidas. As ilustrações das microestruturas foram feitas em fotomicroscópio (MO).

Para o exame da superfície das estruturas fúngicas em microscopia eletrônica de varredura (MEV), a metodologia adotada foi de França \& Sotão (2009). As imagens foram obtidas em MEV marca LEO, modelo 1450VP e registradas em modo digital.

Literatura especializada com descrições de espécies e chaves de identificação como: Cummins \& Hiratsuka (2003), Hennen et al. (2005), Ono (1984) e Carvalho Jr. \& Hennen (2009), foi utilizada para identificação, descrição da espécie e na elaboração da chave de identificação proposta.

As amostras foram depositadas no Herbário João Murça Pires (MG), do Museu Paraense Emílio Goeldi, Belém (PA).

\section{Resultados e Discussão}

A espécie M. bolivarensis coletada na Flona do Amapá, parasitando planta do gênero Manilkara (Sapotaceae) representa o primeiro registro da espécie para o Brasil. Anteriormente esta espécie era conhecida somente pelo espécime tipo coletado sobre Manilkara zapota (L.) P. Royen (como Achras zapota L.) na Venezuela. Sobre Manilkara também foi indentificado para a área de estudo a espécie M. sapotae, sendo um novo registro para o Estado do Amapá.

Maravalia bolivarensis Y. Ono, Mycologia 76(5): 904 (1984).

Figura $1 \mathrm{a}-\mathrm{c}$ 


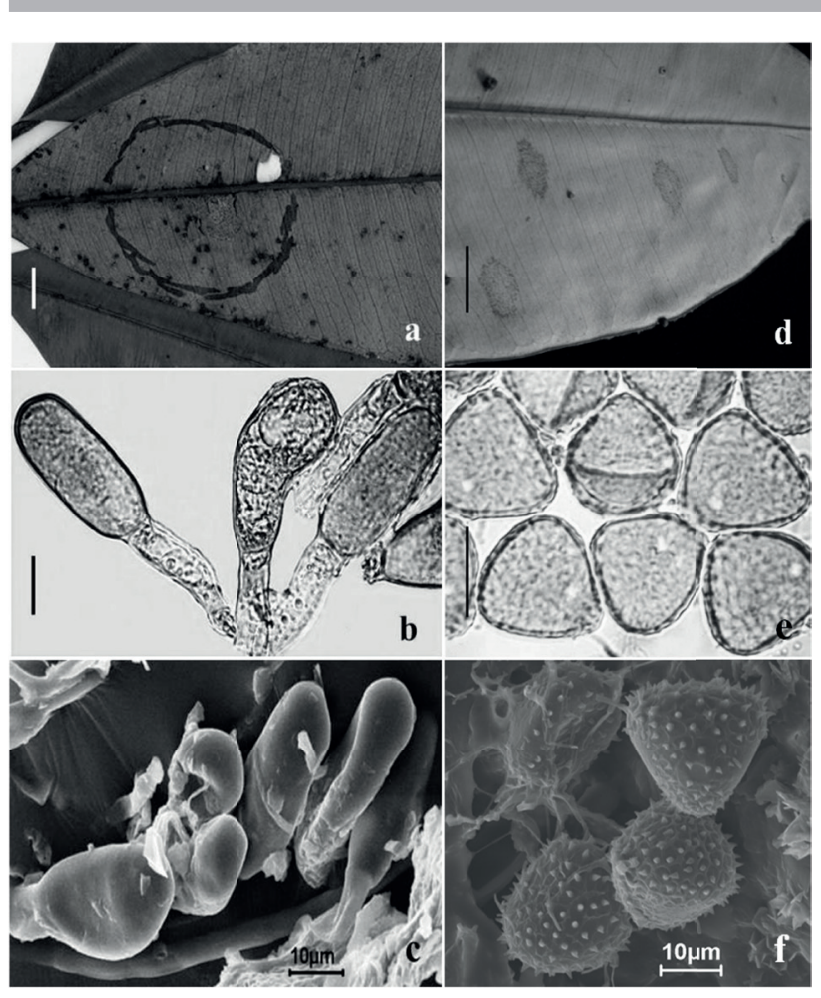

Figura 1. a-c. Manchas foliares e esporos de Maravalia bolivarensis Y. Ono, nova referência para o Brasil. d-f. Manchas foliares e esporos de Maravalia sapotae (Mains) Y. Ono nova referência para o Estado do Amapá. a. Soros de M. bolivarensis em folha de Manilkara sp. b. Teliósporos observados em microscópio óptico de luz (MO). c. Teliósporos observados em microscopia eletrônica de varredura (MEV). d. Soros de M. sapotae em folha de Manilkara sp. e. Urediniósporos observados em microscópio óptico de luz (MO). f. Urediniósporos observados em microscopia eletrônica de varredura (MEV). Barras $=10 \mathrm{~mm}(\mathrm{a}, \mathrm{d}) ; 10 \mu \mathrm{m}$ (b, c, f); $20 \mu \mathrm{m}$ (e).

Figure 1. a-c. Leaf spots and spores of Maravalia bolivarensis Y. Ono, a new record for Brazil. d-f. Leaf spots and spores of Maravalia sapotae (Mains) Y. Ono, a new record for Amapá State. a. Sori of M. bolivarensis on the leaf of Manilkara sp. b. Teliospores observed under optical light microscope (OM). c. Teliospores observed under scanning electron microscope (SEM). d. Sori of M. sapotae on the leaf of Manilkara sp. e. Urediniospores observed under optical light microscope (OM). f. Urediniospores observed under scanning electron microscope $(\mathrm{SEM})$. Bars $=10 \mathrm{~mm}(\mathrm{a}, \mathrm{d}) ; 10 \mu \mathrm{m}(\mathrm{b}, \mathrm{c}, \mathrm{f}) ; 20 \mu \mathrm{m}(\mathrm{e})$.

Holótipo: VENEZUELA. BolIVAR: San Rafael, sobre Manilkara zapota (L.) P. Royen (como Achras zapota L.), 7-VIII-1972, K.P. Dumont, R.F. Samuel e C. Blanco VE7263 (PUR).

Espermogônio, écio e uredínio não observados. Télios abaxiais, agregados, confluentes, pulverulentos, dispersos ou agrupados em manchas ou pústulas, subepidermais em origem, posteriormente irrompentes, com a epiderme circundando o soro; teliósporos de uma célula, pedicelados, elipsóides, oblongos elipsóides a cilíndricos, arredondados no ápice, atenuados na base, $39-35 \times 22-19 \mu \mathrm{m}$, marrom claro, lisos, parede uniforme 1-1,5 $\mu \mathrm{m}$ de espesura, poros germinativos imperceptíveis, apresentam pedicelo persistente e higroscópico (19-25 $\mu \mathrm{m})$.

Material estudado: BRASIL. AmAPÁ: Ferreira Gomes, Flona do Amapá, sobre Manilkara sp., 27-V-2011, H. Sotão (MG214262).

Distribuição: Venezuela (Ono 1984); Brasil, primeiro registro de ocorrência neste estudo.

As características dos teliósporos observadas no espécime examinado estão de acordo com a descrição de Ono (1984). A espécie se diferencia das demais, que ocorrem sobre plantas da família Sapotaceae, principalmente por apresentar teliósporos alongados (39-35 × 22-19 $\mu \mathrm{m})$, com pedicelos higroscópicos e ausência de urediniósporos.

Das onze espécies de Maravalia que ocorrem sobre plantas da família Sapotaceae, somente $M$. bolivarensis não apresenta estádios anamorfos conhecidos e é, provavelmente, uma espécie microcíclica.

Hernández et al. (2005) descrevendo Uredo baruensis J.R. Hern. \& Aime como nova espécie sobre Chrysophyllum sparsiflorum Klotzsch ex Miq. (Sapotaceae) para a Guiana, sugerem que esta espécie seja um anamorfo do gênero Maravalia, porém é distinta dos urediniósporos já descritos para as espécies conhecidas em Sapotaceae. A espécie da planta hospedeira C. sparsiflorum tem distribuição conhecida no Brasil para vários Estados do bioma Amazônia, para o Centro Oeste e Nordeste (Flora do Brasil 2020 em construção 2017), e devido à área de fronteira da Guiana com a Amazônia brasileira, os autores supõem que provavelmente esta espécie de ferrugem também ocorra em plantas desta espécie no Brasil.

Maravalia sapotae (Mains) Y. Ono, Mycologia 76(5): 908 (1984).

Figura $1 \mathrm{~d}-\mathrm{f}$

Literatura com descrição e ilustração: Ono (1984), Sotão et al. (2013).

Material estudado: BRASIL. AmAPÁ: Porto Grande, Flona do Amapá, sobre Manilkara sp., 4-VI-2010, H. Sotão et al. (MG214263); ibid., 13-XI-2011, R. Furtado (MG 207856); ibid., 14-XI-2011, H. Sotão (MG207860). 
Distribuição: Bahamas, Belize, Bermuda, Cuba e Venezuela (Ono 1984), e para o Brasil para a Flona de Caxiuanã, no Estado do Pará (Sotão et. al. 2013).

Esta espécie possui como característica marcante urediniósporos em formatos semitriangulares, de coloração amarelo-escuro ou marrom-escuro, equinulados, com áreas irregulares lisas próximas à base; télios cerosos; teliósporos pedicelados, obovóides, amarelo-claros, lisos; pedicelos persistentes, hialinos.

Nas descrições de M. lucumae sobre Lucuma e M. palaquii sobre Palaquium não existe diferenciação morfológica capaz de separar as duas espécies, exceto o gênero dos hospedeiros, conforme evidenciado nas chaves de Ono (1984) e Carvalho Jr. \& Hennen (2009). Hennen et al. (2005), comentam que é necessário a identificação correta do hospedeiro do espécime tipo M. palaquii, pois o gênero Palaquium não tem ocorrência registrada para o Brasil, e sim para Ásia.

A distinção morfológica entre os teliósporos de M. bolivarensis e de outras espécies que ocorrem sobre Sapotaceae no Brasil, são apresentadas na chave dicotômica.

Chave das espécies de Pucciniales com teliósporos presentes sobre plantas da família Sapotaceae no Brasil

1. Teliósporos com células basais assemelhando-se a pedicelo; presença de paráfises no soro Catenulopsora henneneae

1. Teliósporos com pedicelo definido e sem paráfises no soro

2. Teliósporo com pedicelo higroscópico; urediniósporos quando presentes com parede simples

3. Anamorfos ausentes (microcíclica); teliósporos (35-38,5 × 15-17,5 $\mu \mathrm{m})$; sobre Manilkara

Maravalia bolivarensis

3. Anamorfos presentes; teliósporos sobre Lucuma ou Palaquium

4. Sobre Lucuma; probasídios (35-) 38-50 (-54) × (12-) 14-19 $\mu \mathrm{m}$; pedicelos $50 \mu \mathrm{m}$ de comprimento

M. lucumae

4. Sobre Palaquium; probasídios (36-) 41-62 × 9-18 (-19) $\mu \mathrm{m}$; pedicelos 20-55 $\mu \mathrm{m}$ de comprimento .... M.palaquii

2. Teliósporos com pedicelo não higroscópico; urediniósporos quando presentes bilaminados

5. Teliósporos cilíndricos $(66-99 \times 12-15 \mu \mathrm{m})$, parede com 2-4,5 $\mu \mathrm{m}$ de espessura; pedicelos 50-60 $\mu \mathrm{m}$ de comprimento; urediniósporos quando presentes equinulados sem áreas lisas e poros germinativos de $2-4$ espalhados .... Achrotelium lucumae

5. Teliósporos obovoides $(25-38 \times 15-27,5 \mu \mathrm{m})$, parede uniforme $1 \mu \mathrm{m}$ de espessura; pedicelos 18-20 $\mu \mathrm{m}$ de comprimento; urediniósporos quando presentes equinulados com áreas lisas irregulares próximo a base e dois poros germinativos próximo ao hilo

M. sapotae

\section{Agradecimentos}

Os autores agradecem ao MPEG, UFRA, IEPA e ICMBio pela infraestrutura e suporte técnico oferecidos para realização deste trabalho; ao CNPq pelo apoio financeiro concedido pelo Programa de Biodiversidade da Amazônia (PPBio), projetos via edital Universal e SISBIOTA; aos técnicos Luís Carlos Lobato e Mário Rosa dos Santos (MPEG) pelo auxílio de campo e determinação das espécies vegetais; o primeiro autor agradece ao CNPq pela concessão da bolsa de Mestrado.

\section{Literatura citada}

Aime, M.C., Matheny, P.B., Henk, D.A., Frieders, E.M., Nilsson, R.H., Piepenbring, M., McLaughlin, D.J., Szabo, L.J., Begerow, D., Sampaio, J.P., Bauer, R., Weiss, M., Oberwinkler, F. \& Hibbett, D. 2006. An overview of the higher level classification of Pucciniomycotina based on combined analyses of nuclear large and small subunit rDNA sequences. Mycologia 98: 896-905.

Cummins, G.B. \& Hiratsuka, Y. 2003. Illustrated genera of rust fungi. 3 ed. American Phytopathological Society (APS), St. Paul, Minnesota. 
Carvalho Jr., A.A. \& Hennen J.F. 2009. Maravalia perae, a new species of rust fungus on Pera from Brazil. Mycologia 101: 239-242.

Figueiredo, M.B. \& Passador, M.M. 2008. Morfologia, funções dos soros e variações dos ciclos vitais das ferrugens. Arquivo do Instituto Biológico 75: 117-134.

Flora do Brasil 2020 em construção. 2017. Jardim Botânico do Rio de Janeiro. Disponível em http:// floradobrasil.jbrj.gov.br/ (acesso em 25-IV-2017).

França, I.F. \& Sotão, H.M.P. 2009. Novos registros de ferrugens (Uredinales) sobre Fabaceae para o Brasil. Acta Botanica Brasilica 23: 860-863.

Hennen, J.F., Figueiredo, M.B., Carvalho Jr., A.A. \& Hennen, P.G. 2005. Catalogue of plant rust fungi (Uredinales) of Brazil. Disponível em http://www.jbrj. gov.br (acesso em 03-V-2017).

Hernández, J.R., Aime, M.C. \& Henkel, T.W. 2005. The rust fungi (Uredinales) of Guyana. Sydowia 57: 189-222.
IBAMA. 2017. Instituto Brasileiro do Meio Ambiente e dos Recursos Naturais Renováveis. Disponível em http://www.ibama.gov.br (acesso em 20-V-2017).

Neergaard, E., Lyshede, O.B., Gahoonia, T.S., Care, D. \& Hooker, J.E. 2000. Anatomy and histology of roots and root-soil boundary. In: A.L. Smit, A.G. Bengough, C. Engels, M. Noordwijk, S. Pellerin \& S.C. Geijn (eds.). Root Methods: A. Handbook. Springer-Verlag, Berlin, pp. 33-73.

Ono, Y. 1984. A monograph of Maravalia (Uredinales). Mycologia 76: 892-911.

Sotão, H.M.P., Hennen, J.F., Freire, E.S., Mendonça, F., Brito, F., França, I. \& Castro, C. 2013. Novos registros de fungos (Pucciniales) para a Floresta Nacional de Caxiuanã, Amazônia e Brasil. In: P.L.B. Lisboa (orgs.). Caxiuanã: paraíso ainda preservado. Museu Paraense Emílio Goeldi, Belém, pp. 367-374. 